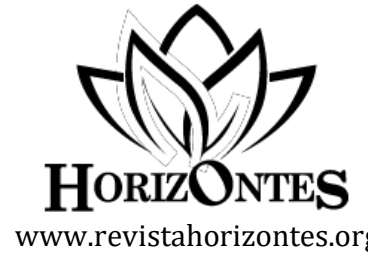

Horizontes. Revista de Investigación en Ciencias de la Educación Https://doi.org/10.33996/revistahorizontes.v4i15.116

Julio - Septiembre 2020

Volumen 4 / No. 15

ISSN: $2616-7964$

ISSN-L: $2616-7964$ pp. $290-296$

\title{
Análisis de carga horaria presencial para alcanzar nivel A1 Inglés. Caso: Carrera Comunicación Social UPSE
}

\author{
Analysis of in-person credit hours class to reach English level A1. Study case: Social \\ Communication Studies, UPSE \\ Análise de horas presenciais para atingir o nível A1 Inglês. Caso: Carreira de \\ Comunicação Social UPSE
}

ARTICULO ORIGINAL

Cecilia Alexandra Jara Escobar

ajara@upse.edu.ec

ORCID: 0000-0001-8282-7017
Rosa Tatiana García Villao
rgarcia@upse.edu.ec
ORCID: 0000-0002-1756-7439

\author{
Herman Christian Zuñiga Muñoz \\ hzuniga@upse.edu.ec \\ ORCID: 0000-0002-5924-4718
}

\author{
Rosa Elena Niola Sanmartín \\ rniola@upse.edu.ec \\ ORCID: 0000-0002-1879-7367
}

\section{Universidad Estatal Península de Santa Elena, Ecuador}

Recibido abril 2020 | Revisado mayo 2020 | Publicado en julio 2020

\section{RESUMEN}

El presente estudio analizó la realidad del proceso enseñanza y aprendizaje del idioma Inglés de los estudiantes de la carrera de Comunicación Social nivel 1, de la Universidad Estatal Península de Santa Elena. El objetivo es demostrar si 32 horas clases presenciales de enseñanza del idioma inglés permiten alcanzar el nivel A1 del idioma de acuerdo al Marco Común Europeo de Referencia para las lenguas, tomando como herramienta principal la metodología de enseñanza de blended learning. El estudio se realizó bajo el planteamiento metodológico mixto, que permite demostrar la hipótesis planteada; el procedimiento se aplicó bajo un diseño longitudinal, acompañado de una metodología descriptiva y cuasi-experimental. Para la recolección de datos se utilizó la encuesta y la documentación. Los resultados obtenidos demuestran la necesidad de incrementar la carga horaria para que los estudiantes logren alcanzar el nivel A1, ya que sus habilidades y destrezas no se comparan con lo exigido por el Marco Común Europeo.

Palabras clave: Carga horaria; clases presenciales, suficiencia del idioma

\section{ABSTRACT}

The present study analyzed the reality of the English language teaching and learning process of the students from the Social Communication career, first semester, classes at Universidad Estatal Península de Santa Elena. The objective was to demonstrate whether 32 hours of face-to face English language teaching allow students to reach level A1 of the language according to the Common European Framework of Reference for Languages, using Blended Learning as the main teaching method. The study was carried out under the mixed methodological approach, which permitted to demonstrate the hypothesis; the procedure was applied under a longitudinal design, accompanied by a descriptive and quasi-experimental methodology. For data collection, a survey and documentation were used. The obtained results demonstrate the need to increase the number of hours of instruction so that students achieve level A1, since their abilities and skills are not the ones required by the Common European Framework.

Key words: face-to-face classes, hours of instruction, language proficiency 


\section{RESUMO}

0 presente estudo analisou a realidade do processo de ensino e aprendizagem da língua inglesa dos alunos do curso de Comunicação Social nível 1, da Universidade Estadual da Península de Santa Elena. 0 objetivo é demonstrar se 32 horas de ensino presencial da língua inglesa permitem atingir o nível A1 da língua de acordo com o Quadro Europeu Comum de Referência para as línguas, utilizando a metodologia de ensino blended learning como principal ferramenta. 0 estudo foi realizado sob a abordagem metodológica mista, o que permite demonstrar a hipótese levantada; o procedimento foi aplicado sob um delineamento longitudinal, acompanhado de uma metodologia descritiva e quase experimental. Para a coleta de dados, foram utilizados o levantamento e a documentação. Os resultados obtidos demonstram a necessidade de aumentar a carga horária para que os alunos atinjam o nível A1, uma vez que as suas capacidades e aptidões não se comparam com o que é exigido pelo Quadro Europeu Comum.

Palavras-chave: Carga horária; aulas presenciais, proficiência no idioma

\section{INTRODUCCIÓN}

El Inglés es considerado un idioma universal y lengua franca en los diversos ámbitos profesionales, comunicacionales, y sociales. Se ha convertido en una lengua internacional que ha repercutido en todos los países no-anglosajones, incluida España, por razones de globalización, negocios, educación, etc. Su aprendizaje ya no puede tratarse como un lujo, es una necesidad evidente (Chávez Zambrano, y Saltos Vivas, 2017).

El dominio del idioma Inglés es considerado requisito obligatorio para trabajar en las instituciones internacionales. En una consulta a 189 países miembros de las Naciones Unidas sobre el idioma deseado para la comunicación entre embajadas, más de 120 seleccionaron el inglés (El Nuevo Diario y Ortiz, 2013).

En la educación superior se espera que los estudiantes dominen ciertas destrezas basadas en el marco Común Europeo de Referencia para las lenguas. Por este motivo las Instituciones de Educación Superior deben garantizar el cumplimiento de un número de horas básicas y la calidad en el proceso de enseñanzaaprendizaje. En Ecuador, tener la suficiencia de este idioma es un requisito para obtener el título de tercer nivel y para ingresar a realizar estudios de posgrado a nivel nacional. El Reglamento de Régimen Académico en el Ecuador, Artículo 80, señala que es requisito de graduación en las carreras de tercer nivel el dominio de una lengua extranjera, que corresponda al nivel B1 del Marco Común Europeo de Referencia (MCER) para las lenguas. Consejo de Educación Superior, (2019).

El B-learning o Blended Learning es una modalidad semipresencial que ayuda en el proceso de aprendizaje de los estudiantes en un ambiente de aprendizaje combinado B-learning, en el que se mezcla diferentes metodologías incluyendo las clases presenciales, así como las clases no presenciales, en la que los estudiantes pueden reforzar $\mathrm{y}$ ejercitar de manera autónoma los contenidos revisados en clases y a la vez utilizar herramientas de comunicación y de trabajo colaborativo como aporte para el desarrollo de la comprensión oral del idioma inglés (Andrade-Velásquez, 2015).

Morrison (2003) recomienda que el Aprendizaje Combinado se utilice como una estrategia de enseñanza para seleccionar los canales de transmisión del conocimiento. Es por ello que también es necesario establecer qué elementos de la educación a distancia serían los mejores para cumplir los objetivos y qué porcentaje de las sesiones se dedicarán a la presencialidad. La definición más sencilla y precisa describe al aprendizaje combinado como aquel modo de aprender que combina la enseñanza presencial con la tecnología no presencial, (Coaten, 2003; Marsh et al., 2003).

Para que un individuo alcance la suficiencia en los diferentes niveles de aprendizaje de un 
idioma extranjero, Cambridge (2019) propone que, para lograr un nivel $\mathrm{A} 1$, principiante, se necesita entre 100 y 140 horas de dedicación. Según Archibald et al. (2006) 5000 horas de instrucción es insuficiente para que los estudiantes alcancen el bilingüismo funcional. El presente artículo analiza la situación de los estudiantes de la carrera de Comunicación Social de la Universidad Estatal Península de Santa Elena (UPSE) a quienes se les ha asignado 32 (treinta y dos) horas de clases presenciales en el sistema de créditos de la asignatura de inglés. Por lo tanto, la hipótesis a comprobar en este artículo es ¿son suficientes 32 horas de clases presenciales para alcanzar el nivel A1 en el idioma Inglés? Cummins (1981) acotó que incluso a los niños que han estado inmersos $100 \%$ en un idioma, les podría tomar años alcanzar altos niveles de dominio.

El estudio tuvo como objetivo principal comprobar si la asignación de 32 horas de clases presenciales de aprendizaje del idioma inglés permite alcanzar el nivel $\mathrm{A} 1$, cuyos lineamientos constan en el Marco Común Europeo de Referencia para las Lenguas. Los objetivos específicos que acompañan al mismo son: a. Demostrar el nivel de aprendizaje que se logró en los estudiantes de Comunicación Social. b. Documentar en un journal la experiencia de los estudiantes con respecto al aprendizaje del idioma inglés en la etapa inicial del tratamiento. c. Analizar los resultados del proceso de enseñanza aprendizaje durante el tratamiento en términos de contenidos revisados del sílabo.

\section{MÉTODO}

Este estudio se realizó bajo el planteamiento metodológico mixto, que permitió demostrar la hipótesis planteada; el procedimiento se aplicó bajo un diseño longitudinal, acompañado de una metodología descriptiva y cuasi-experimental. El diseño longitudinal permitió comprobar resultados al inicio y al final del presente estudio. La metodología descriptiva permitió identificar las características y propiedades del grupo de estudio; mientras que el sistema cuasiexperimental permitió la aplicación y respuesta del proceso de estudio, e incluyó sujetos asignados de manera no aleatoria a los docentes involucrados; además, es el más aplicable al área educativa que corresponde a las ciencias sociales.

En este sentido, la población de este estudio estuvo integrada por un grupo de tratamiento, que permitió medir y comprobar las variables del mismo. Al ser un diseño longitudinal, el estudio fue realizado durante 16 semanas que corresponden al semestre 2019-I; para esto se realizó un test de diagnóstico o pre-test y un test final o post-test, con los dos grupos asignados. La población fue seleccionada por asignación a los docentes involucrados en este estudio. Al contar con dos cursos con similares características como la carrera universitaria, número de estudiantes, número de horas de la asignatura, edad, y horario de clases; se determinó que la muestra serían los estudiantes de la carrera de Comunicación Social, del primer semestre, del período académico 20182 , que constaba de 66 estudiantes en total, en la asignatura de Inglés I.

La técnica de recolección de datos que se utilizó para el presente estudio es la encuesta y la documentación. Para la recolección de datos a través de una encuesta, se aplicaron dos cuestionarios de conocimientos del idioma inglés denominado prueba de diagnóstico, al inicio del estudio y examen final, al concluir el tratamiento. Los cuestionarios constaron de una estructura de 40 preguntas distribuidas para medir las destrezas de lectura, escritura, habla y escucha, además conocimiento de vocabulario y gramática. Los resultados fueron analizados y se determinó la validez de la 
hipótesis. La documentación se realizó a través de un journal en donde se registraron las actividades y experiencias del docente durante el tratamiento.

\section{RESULTADOS}

Durante el semestre 2019-I, en la Carrera de Comunicación en la asignatura Inglés 1 , se designaron 2 horas presenciales a la semana para la enseñanza del idioma, los docentes emplearon la metodología de Blended Learning, la misma que consistía en el desarrollo de actividades de carácter autónomo y colaborativo para poder alcanzar los objetivos de este módulo.

Durante el periodo de enseñanza de este estudio se realizaron actividades como Role play, concursos de verbos, pequeños escritos básicos, trabajos en pareja, entre otros. El objetivo de las actividades presenciales era principalmente practicar la producción y comprensión oral, además de explicar los aspectos léxicos y sintácticos de los contenidos. Particularmente, el enfoque se realizaba en aquellos temas que fueran necesarios para la comprensión y el desarrollo de las actividades on line. Por otra parte, se destacó el trabajo autónomo de los participantes, quienes realizaron trabajos de refuerzo en casa como ejercicios de gramática, lectura, vocabulario, y escritura. Estas actividades contribuyeron a la comprensión y aprendizaje de los contenidos, como parte del proceso del método Blended Learning.

Una vez realizado el tratamiento con el grupo de participantes, con el fin de determinar el alcance del nivel básico de inglés $\mathrm{A} 1$, se analizaron los datos obtenidos, a través de la técnica de encuesta, tanto de la prueba de diagnóstico y la evaluación final.

En la Figura 1 se muestran los resultados alcanzados en la prueba de diagnóstico, además se visualizan los datos obtenidos en esta evaluación inicial. (Ver Figura 1).

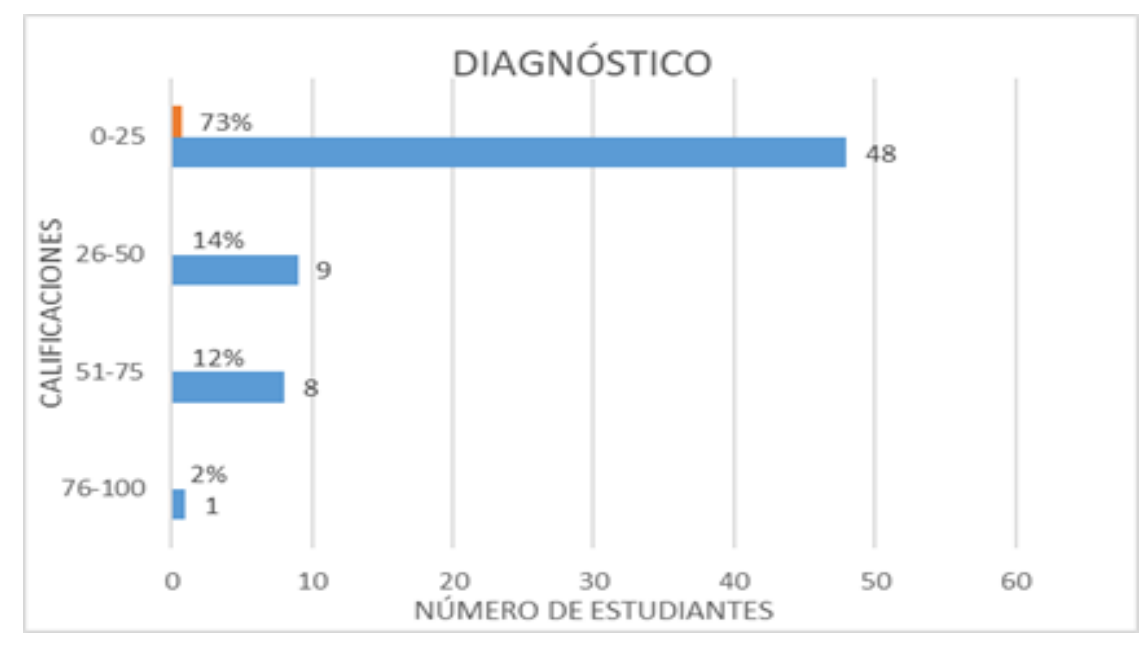

Figura 1. Resultados Prueba de diagnóstico

El 98\% de los estudiantes de estos dos paralelos presentaron resultados inferiores a $76 / 100$ en las pruebas de diagnóstico. El 14\% obtuvo una calificación entre 26 y 50 puntos.
Mientras que el 12\% de los resultados alcanzó un puntaje entre 51 y 75 puntos. Solamente el $2 \%$ logró una nota superior, entre 76 y 100 puntos. 
El progreso de los participantes ha sido monitoreado constantemente a través de diferentes tipos de evaluación que formaron parte del sílabo, e incluían parámetros de evaluación formativa y sumativa como: tareas y actividades, exámenes de primero y segundo parcial, que al finalizar el estudio determinaron el avance académico de los estudiantes.

Una vez concluido el tratamiento, se realizó la evaluación final cuyos resultados son representados en la Figura 2 que guarda relación con el Promedio Final.

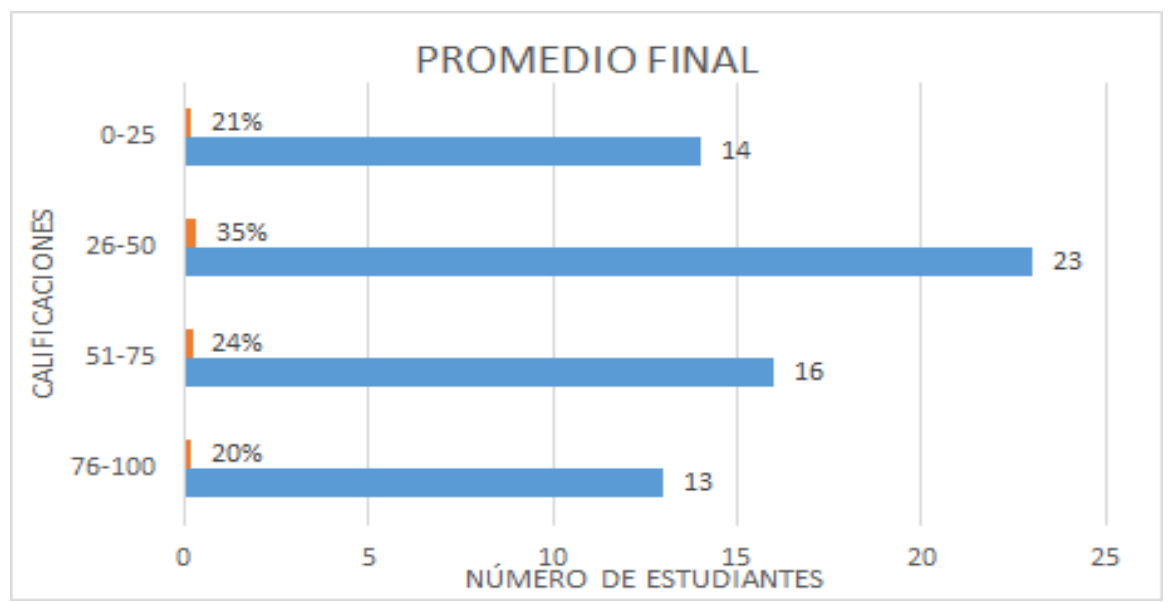

Figura 2. Promedio Evaluación final

De acuerdo con estos datos, se ha podido evidenciar que solamente el $20 \%$ aprobó la asignatura con una nota superior a 76/100. Sin embargo, se ha registrado que el $35 \%$ de los participantes mejoró sus conocimientos y habilidades en el idioma. Además un 24\% obtuvo una calificación entre 51 y 75 puntos. Finalmente, el $21 \%$ no ha evidenciado progreso en su aprendizaje.

\section{Discusión}

Al finalizar el estudio es posible establecer una comparación entre la propuesta de la carga horaria de Cambridge, que sugiere entre 100 a 140 horas para alcanzar el nivel A1 en el idioma inglés y la situación de la carrera de Comunicación Social, UPSE, que al momento del estudio contaba con una asignación de 32 horas presenciales en el sistema de créditos de la asignatura de Inglés I.

Uno de los objetivos planteados en este estudio ha sido comprobar si la asignación de
32 horas de clases presenciales permiten alcanzar el nivel A1 de Inglés, el mismo que está en concordancia con el objetivo específico de demostrar el nivel de aprendizaje del idioma Inglés alcanzado por los estudiantes de la carrera de Comunicación Social, evidentemente los resultados del examen de diagnóstico y la evaluación final demostraron un avance en el desarrollo de las destrezas del idioma; así al inicio del estudio $73 \%$ de estudiantes alcanzaron entre 0 y 25 puntos, este número fue reducido a $21 \%$ de estudiantes en el mismo rango de notas al final del estudio. Así mismo, al inicio solamente un $2 \%$ de los participantes lograron un puntaje entre 67 y 100 puntos, mientras que al final del mismo se observó un progreso del $20 \%$ en el mismo rango. Sin embargo, este progreso no cumple con las especificaciones del MCER, ya que se encuentran limitaciones en la práctica de habilidades como lectura comprensiva, escritura, escucha y habla, para aprobar la 
asignatura, los estudiantes deben alcanzar un mínimo de 70/100 puntos.

De acuerdo a la información recabada por los docentes, en el journal, al inicio del tratamiento, se manifiesta que cuentan con un grupo variado de participantes en los siguientes contextos: algunos participantes demostraron tener conocimientos muy básicos del idioma inglés, otros jamás tuvieron oportunidad de estudiar, mientras que, otros recordaban pocas palabras aprendidas en su educación primaria y/o secundaria, y otro grupo de participantes tenía la idea preconcebida que el inglés es un idioma difícil o causa poco interés.

Se analizaron también los resultados del proceso de enseñanza aprendizaje en el presente estudio, en términos de los contenidos revisados del sílabo, los mismos que, debido a la carga horaria, fueron reducidos y adaptados a la realidad del curso; en el programa constaban 5 unidades de aprendizaje con 4 temas en cada una, las mismas se mantuvieron en número, pero se redujeron a 2 temas por cada unidad. De esta manera, los resultados de aprendizaje fueron medianamente cumplidos.

\section{CONCLUSIONES}

Al finalizar este estudio, se concluyó que a pesar de los resultados de los estudiantes que aprobaron el curso, éstos no dominan los contenidos correspondientes al nivel A1 del Marco Común Europeo de Referencia para las Lenguas, demostrando que el número de horas no fue suficiente para el aprendizaje $y$ desarrollo de las destrezas de este nivel.

Las 32 horas de carga horaria de clases presenciales de aprendizaje del idioma inglés solicitada por la carrera de Comunicación en comparación a las 140 horas que indica el MCERL, no son suficiente para alcanzar el nivel A1, los docentes llegan a cumplir metas medianamente satisfactorias, pues deben distribuir el programa entre el desarrollo de las cuatro destrezas y los temas de gramática; se evidencia que estos aspectos no se cumplen al $100 \%$.

Es necesario analizar aspectos como el reajuste del currículo así como de la carga horaria adecuada para el aprendizaje de una lengua extranjera, la misma que debe ser establecida bajo los parámetros del MCER y las autoridades y docentes involucrados en el proceso de enseñanza.

La metodología de enseñanza usada en este tratamiento, Blended Learning, contribuye al aprendizaje de los participantes, siempre y cuando se utilicen los recursos, materiales, actividades, etc. como el enfoque de estudio de una lengua. Es importante, además, el tiempo de dedicación que se preste al aprendizaje autónomo ya que determina el alcance de los objetivos del programa de estudio. La documentación en el journal refleja el nivel de conocimiento y percepciones que tienen los estudiantes sobre el idioma inglés.

\section{REFERENCIAS}

Archibald, J., Roy, S., Harmel, S., Jesney, K., Dewey, E., Moisik, S., et al. (2006). A review of the literature on second language learning (2nd ed.): Alberta Education

Cambridge English, 2016. The Cambridge English Scale. Cambridge English Language Assessment, (CB1 2EU), pp.3-5

CES, 2019. Reglamento Académico de Educación Superior. (RPC-SO-08-No.1112019), pp.31 de 53

Chávez Zambrano, M., y Saltos Vivas, M. (2017, agosto 22). La importancia del aprendizaje y conocimiento del idioma Inglés en la enseñanza superior. Revista Científica Dominio de las Ciencias, 3(2), 759-771. DXDOI.org/10.23857/dom.cien.pocaip.201 7.3.mono1.ag0.759-771

COATEN, N. (2003) Blended e-learning. Educaweb, 69. [Online, revisado 20 julio, 2005] http://www.educaweb.com/esp /servicios/ monografico/formacionvirtual /1181076.asp 
Cummins, J. (1981). The role of primary language development in promoting educational success for language minority students. In Schooling and language minority students: A theoretical framework. Los Ángeles: UCLA

El Nuevo Diario y Ortiz, I. (2013, mayo 30). Importancia del idioma Inglés en la Educación. La importancia del idioma inglés en la Educación

Graham, C. R. (2006). Blended Learning Systems. Definition, current trends and
Future Directions. En J. Curtis, Ch. Bonk y R. Graham (Ed.), The Handbook of Blended Learning: Global Perspectives, Local Designs. John Wiley y Sons

Morrison, Don. 2003. E-learning strategies: How to get implementation and delivery right the first time. New York: John Wiley

Velásquez, M. A. (2015). Desarrollo de la comprensión oral del idioma inglés a través del aprendizaje combinado B-learning en estudiantes universitarios. Universitas, (23), 109-124 